\title{
Double Diffusive Convection in a Layer of Maxwell Viscoelastic Fluid in Porous Medium in the Presence of Soret and Dufour Effects
}

\author{
Ramesh Chand ${ }^{1}$ and G. C. Rana ${ }^{2}$ \\ ${ }^{1}$ Department of Mathematics, Government College Dhaliara, Himachal Pradesh 177103, India \\ ${ }^{2}$ Department of Mathematics, Government College Nadaun, Himachal Pradesh 177033, India \\ Correspondence should be addressed to Ramesh Chand; rameshnahan@yahoo.com
}

Received 7 February 2014; Revised 15 May 2014; Accepted 30 June 2014; Published 17 July 2014

Academic Editor: Mahmoud Mamou

Copyright (C) 2014 R. Chand and G. C. Rana. This is an open access article distributed under the Creative Commons Attribution License, which permits unrestricted use, distribution, and reproduction in any medium, provided the original work is properly cited.

Double diffusive convection in a horizontal layer of Maxwell viscoelastic fluid in a porous medium in the presence of temperature gradient (Soret effects) and concentration gradient (Dufour effects) is investigated. For the porous medium Darcy model is considered. A linear stability analysis based upon normal mode technique is used to study the onset of instabilities of the Maxwell viscolastic fluid layer confined between two free-free boundaries. Rayleigh number on the onset of stationary and oscillatory convection has been derived and graphs have been plotted to study the effects of the Dufour parameter, Soret parameter, Lewis number, and solutal Rayleigh number on stationary convection.

\section{Introduction}

Bénard convection originated from the experimental works of Bénard [1] and theoretical analysis of Lord Rayleigh [2]. Lord Rayleigh studied the dynamic origins of convective cells and proposed his theory on the buoyancy driven convection. The detailed study of Bénard convection in Newtonian fluid in nonporous medium under varying assumptions of hydrodynamics and hydromagnetics had been given by Chandrasekhar [3]. Lapwood [4] had studied the stability of convective flow in hydromagnetics in a porous medium using Rayleigh's procedure. The Rayleigh instability of a thermal boundary layer in flow through a porous medium had been considered by Wooding [5]. McDonnel [6] suggested the importance of porosity in the astrophysical context.

Double-diffusive convection is referred to buoyancydriven flows induced by combined temperature and concentration gradients. The onset of double diffusive convection in a fluid saturated in porous medium is regarded as a classical problem due to its wide range of applications in many engineering fields such as evaporative cooling of high temperature systems, agricultural product storage, soil sciences, enhanced oil recovery, packed-bed catalytic reactors, and the pollutant transport in underground. A detailed review of the literature concerning double diffusive convection in binary fluid in a porous medium was given by Nield and Bejan [7], Trevisan and Bejan [8], and Malashetty and Kollur [9]. Thermal convection in binary fluid driven by the Soret and Dufour effects had been investigated by Knobloch [10] and showed that the equations were identical to the thermosolutal problem except relation between the thermal and solutal Rayleigh numbers. The above literature dealt with Newtonian fluids.

The study of natural convection of non-Newtonian fluids in a porous medium had gained much attention because of its engineering and industrial applications. These applications included design of chemical processing equipment, formation and dispersion of fog, distributions of temperature and moisture over agricultural fields and groves of fruit trees, and damage of crops due to freezing and pollution of the environment.

The fluids that show distinct deviation from "Newtonian hypothesis" (stress on fluid is linearly proportional to strain 
rate of fluid) are called non-Newtonian fluids. Different models had been proposed to explain the behavior of nonNewtonian fluids. Maxwell model is one of them. These fluids help us to understand the wide variety of fluids that exist in the physical world and characterized by power-law model. The work on viscoelastic fluid appears to be that of Herbert on plane coquette flow heated from below. $\mathrm{He}$ found a finite elastic stress in the undistributed state to be required for the elasticity to affect the stability. Using a three constants rheological model due to Oldroyd [11], the author demonstrated, for finite rate of strain, that the elasticity has a destabilizing effect, which results solely from the change in apparent viscosity.

The importance of the study of viscoelastic fluids in a porous medium has been increasing for the last few years. This is mainly due to their applications in petroleum drilling, manufacturing of foods and paper, and many others. The problem of convective instability of viscoelastic fluid heated from below was first studied by Green [12]. Vest and Arpaci [13] investigated problems of overstability in a horizontal layer of a viscoelastic fluid heated from below. Bhatia and Steiner [14] studied the problem of thermal instability of a Maxwellian viscoelastic fluid in the presence of rotation and found that rotation has a destabilizing influence in contrast to its stabilizing effect on a viscous Newtonian fluid. Bhatia and Steiner [15] have also studied the thermal instability of a Maxwellian viscoelastic fluid in hydromagnetic and found that magnetic field had stabilizing effect on the Maxwell fluid, just as in the case of Newtonian fluid. Sharma and Kumar [16] studied the Hall effect on thermosolutal instability in a Maxwellian viscoelastic fluid and found that Hall effect destabilizes the fluid layer while Kirti and Chand [17] studied the combined effect Hall Current, suspended particles, and variable gravity in a layer of Maxwell viscoelastic fluid. Chand and Kango [18], Chand [19-21], and Chand and Kumar [22] studied problems of thermal instability of Maxwell viscoelastic fluid in porous medium under various assumptions. Chand and Rana [23] investigated the Dufour and Soret effects in layer of elasticoviscous fluid in a porous medium and found that Dufour parameter destabilizes the fluid layer while Soret parameter has both the stabilizing and destabilizing effects on fluid layer depending upon certain conditions.

In this paper an attempt has been made to study the Dufour and Soret effects on the onset of instability in a horizontal layer of Maxwell viscoelastic fluid in a porous medium.

\section{Mathematical Formulations of the Problem}

Consider an infinite horizontal layer of Maxwell viscoelastic fluid of thickness " $d$," confined between the planes $z=0$ and $z=d$ in a porous medium of porosity $\varepsilon$ and medium permeability $k_{1}$ and is acted upon by gravity $\mathbf{g}(0,0,-g)$. This layer of fluid is heated and soluted in such a way that a constant temperature and concentration distribution is prescribed at the boundaries of the fluid layer. The temperature $(T)$ and concentration $(C)$ are taken to be $T_{0}$ and $C_{0}$ at $z=0$ and
$T_{1}$ and $C_{1}$ at $z=d,\left(T_{0}>T_{1}, C_{0}>C_{1}\right)$. Let $\Delta T$ and $\Delta C$ be the difference in temperature and concentration across the boundaries.

Let $\mathbf{q}(u, v, w), p, \rho, T, C, \alpha, \alpha^{\prime}, \mu, \kappa$, and $\kappa^{\prime}$ be the Darcy velocity vector, hydrostatic pressure, density, temperature, solute concentration, coefficient of thermal expansion, an analogous solvent coefficient of expansion, viscosity, thermal diffusivity, and solute diffusivity of fluid, respectively.

2.1. Assumptions. The mathematical equations describing the physical model are based upon the following assumptions.

(i) Thermophysical properties expect for density in the buoyancy force (Boussinesq hypothesis) are constant.

(ii) Darcy's model with time derivative is employed for the momentum equation.

(iii) The porous medium is assumed to be isotropic and homogeneous.

(iv) No chemical reaction takes place in a layer of fluid.

(v) The fluid and solid matrix are in thermal equilibrium state.

(vi) Radiation heat transfer between the sides of the wall is negligible when compared with other modes of the heat transfer.

2.2. Governing Equations. According to the works of Bhatia and Steiner [14, 15], Sharma and Kumar [16], and Chand [19-21] the appropriate governing equations for Maxwell viscoelastic fluid in a porous medium are

$$
\begin{aligned}
& \nabla \cdot \mathbf{q}=0, \\
& 0=\left(1+\lambda \frac{\partial}{\partial t}\right) \\
& \times\left(-\nabla p+\rho\left(1-\alpha\left(T-T_{0}\right)-\alpha^{\prime}\left(C-C_{0}\right)\right) \mathbf{g}\right)-\frac{\mu}{k_{1}} \mathbf{q}, \\
& \sigma \frac{\partial T}{\partial t}+\mathbf{q} \cdot \nabla T=\kappa \nabla^{2} T+D_{T C} \nabla^{2} C, \\
& \varepsilon \frac{\partial C}{\partial t}+\mathbf{q} \cdot \nabla C=\kappa^{\prime} \nabla^{2} C+D_{C T} \nabla^{2} T,
\end{aligned}
$$

where $D_{T C}$ and $D_{C T}$ are the Dufour and Soret coefficients; $\sigma=\left(\rho c_{p}\right)_{m} /\left(\rho c_{p}\right)_{f}$ is thermal capacity ratio, $c_{p}$ is specific heat, and the subscripts $m$ and $f$ refer to porous medium and fluid, respectively.

We assume that temperature and concentration are constant at the boundaries of the fluid layer. Therefore, boundary conditions are

$$
\begin{array}{llll}
w=0, & T=T_{0}, & C=C_{0} & \text { at } z=0, \\
w=0, & T=T_{1}, & C=C_{1} & \text { at } z=d .
\end{array}
$$


2.3. Steady State and Its Solutions. The steady state is given by

$$
u=v=w=0, \quad p=p(z), \quad T=T_{s}(z), \quad C=C_{s}(z) .
$$

The solution of steady state is given as

$$
\begin{aligned}
& T_{s}=T_{0}-\frac{\Delta T}{d} z, \\
& C_{s}=C_{0}-\frac{\Delta C}{d} z, \\
& p_{s}=p_{0}-\rho_{0} g\left(z+\alpha \frac{\Delta T}{2 d} z^{2}+\alpha^{\prime} \frac{\Delta C}{2 d} z^{2}\right),
\end{aligned}
$$

where subscript 0 shows the value of the variable at boundary $z=0$.

2.4. Perturbation Solution. To study the stability of the system, we superimposed infinitesimal perturbations on the basic state, which are of the forms

$$
\begin{aligned}
q=0+q^{\prime}, \quad T & =T_{s}+T^{\prime}, \quad C=C_{s}+C^{\prime}, \\
p & =p_{s}+p^{\prime},
\end{aligned}
$$

where the prime denotes the perturbed quantities. Substituting (5) into (1) and neglecting higher order terms of the perturbed quantities, we get

$$
\begin{aligned}
& \nabla \cdot q^{\prime}=0 \\
& 0=\left(1+\lambda \frac{\partial}{\partial t}\right)\left(-\nabla p^{\prime}+\rho_{0}\left(\alpha T^{\prime}+\alpha^{\prime} C^{\prime}\right) \mathbf{g}\right)-\frac{\mu}{k_{1}} \mathbf{q}^{\prime}, \\
& \sigma \frac{\partial T^{\prime}}{\partial t}-w^{\prime} \frac{\Delta T}{d}=\kappa \nabla^{2} T^{\prime}+D_{T C} \nabla^{2} C^{\prime}, \\
& \varepsilon \frac{\partial C^{\prime}}{\partial t}-w^{\prime} \frac{\Delta C}{d}=\kappa^{\prime} \nabla^{2} C^{\prime}+D_{C T} \nabla^{2} T^{\prime} .
\end{aligned}
$$

Introduce the dimensionless variables as

$$
\begin{aligned}
& \left(x^{\prime \prime}, y^{\prime \prime}, z^{\prime \prime}\right)=\left(\frac{x^{\prime}, y^{\prime}, z^{\prime}}{d}\right), \\
& \left(u^{\prime \prime}, v^{\prime \prime}, w^{\prime \prime},\right)=\left(\frac{u^{\prime}, v^{\prime}, w^{\prime}}{\kappa}\right) d, \\
& t^{\prime \prime}=\frac{\kappa}{\sigma d^{2}} t, \quad p^{\prime \prime}=\frac{k_{1} d^{2}}{\mu \kappa} p^{\prime}, \\
& T^{\prime \prime}=\frac{T^{\prime}}{\Delta T}, \quad C^{\prime \prime}=\frac{C^{\prime}}{\Delta C} .
\end{aligned}
$$

Thereafter drop the dashes $\left({ }^{\prime \prime}\right)$ for simplicity.
Equation (6) in nondimensional form can be written as

$$
\nabla \cdot \mathbf{q}=0
$$

$$
0=\left(1+F \frac{\partial}{\partial t}\right)(-\nabla p+\mathrm{Ra} T+\mathrm{Rs} C)-\mathbf{q}
$$

$$
\begin{gathered}
\frac{\partial T}{\partial t}-w=\nabla^{2} T+D_{f} \nabla^{2} C, \\
\frac{\varepsilon}{\sigma} \frac{\partial C}{\partial t}-w=\frac{1}{\mathrm{Le}} \nabla^{2} C+S_{r} \nabla^{2} T .
\end{gathered}
$$

Here nondimensional parameters are as follows.

$\mathrm{Ra}=g \rho_{0} \alpha k_{1} \Delta T d / \mu \kappa$ is the thermal Rayleigh number, Rs $=g \rho_{0} \alpha^{\prime} k_{1} \Delta C d / \mu \kappa^{\prime}$ is the solutal Rayleigh number, Le $=\kappa / \kappa^{\prime}$ is the Lewis number, $F=\left(\kappa / \sigma d^{2}\right) \lambda$ is the stress relaxation parameter, $D_{f}=D_{T C} \Delta C / \kappa \Delta T$ is the Dufour parameter, and $S_{r}=D_{C T} \Delta T / \kappa \Delta C$ is the Soret parameter.

The nondimensional boundary conditions are

$$
w=T=C=0 \quad \text { at } z=0, z=1 .
$$

\section{Normal Modes and Stability Analysis}

Analyze the disturbances into the normal modes and assume that the perturbed quantities are of the form

$$
[w, T, C]=[W(z), \Theta(z), \Gamma(z)] \exp \left(i k_{x} x+i k_{y} y+n t\right)
$$

where $k_{x}, k_{y}$ are wave numbers along $x$ and $y$ directions, respectively, and $n$ is growth rate of disturbances.

Using (10), (8) becomes

$$
\begin{gathered}
\left(D^{2}-a^{2}\right) W+(1+F n)\left(a^{2} \operatorname{Ra} \Theta+a^{2} \operatorname{Rs} \Gamma\right)=0, \\
W+\left(D^{2}-a^{2}-n\right) \Theta+D_{f}\left(D^{2}-a^{2}\right) \Gamma=0, \\
W+S_{r}\left(D^{2}-a^{2}\right) \Theta+\left(\frac{1}{\operatorname{Le}}\left(D^{2}-a^{2}\right)-\frac{\varepsilon}{\sigma} n\right) \Gamma=0,
\end{gathered}
$$

where $D=d / d z$ and $a=\sqrt{k_{x}^{2}+k_{y}^{2}}$ is the dimensionless resultant wave number.

The boundary conditions are

$$
\begin{aligned}
& W=0, \quad D^{2} W=0, \quad \Theta=0, \quad \Gamma=0 \quad \text { at } z=0, \\
& W=0, \quad D^{2} W=0, \quad \Theta=0, \quad \Gamma=0 \quad \text { at } z=1 .
\end{aligned}
$$

We assume the solution to $W, \Theta$, and $\Gamma$ is of the form

$$
W=W_{0} \sin \pi z, \quad \Theta=\Theta_{0} \sin \pi z, \quad \Gamma=\Gamma_{0} \sin \pi z,
$$

which satisfy boundary conditions (12). 
Substituting solution (13) in (11), integrating each equation from $z=0$ to $z=1$ by parts, we obtain the following matrix equation as

$$
\left[\begin{array}{ccc}
J & -a^{2}(1+F n) \mathrm{Ra} & -a^{2}(1+F n) \mathrm{Rs} \\
-1 & (J+n) & D_{f} J \\
-1 & S_{r} J & \left(\frac{J}{\mathrm{Le}}+\frac{\varepsilon n}{\sigma}\right)
\end{array}\right]\left[\begin{array}{c}
W_{0} \\
\Theta_{0} \\
\Gamma_{0}
\end{array}\right]=\left[\begin{array}{l}
0 \\
0 \\
0
\end{array}\right],
$$

where $J=\pi^{2}+a^{2}$.

The nontrivial solution of the above matrix requires that

$$
\begin{aligned}
\mathrm{Ra}= & \frac{\left(J(J+n)((J / \mathrm{Le})+(\varepsilon n / \sigma))-S_{r} D_{f} J^{2}\right)}{a^{2}(1+F n) J\left((1 / \mathrm{Le})-D_{f}\right)+(\varepsilon n / \sigma)} \\
& +\frac{S_{r} J-(J+n)}{J\left((1 / \mathrm{Le})-D_{f}\right)+(\varepsilon n / \sigma)} \mathrm{Rs.}
\end{aligned}
$$

For neutral instability $n=i \omega$, (where $\omega$ is real and dimensionless frequency of oscillation) and equating real and imaginary parts of (15), we have

$$
\begin{aligned}
& J\left(\left(\frac{J^{2}}{\mathrm{Le}}-\frac{\omega^{2} \varepsilon}{\sigma}\right)-S_{r} D_{f} J^{2}\right)+a^{2} \operatorname{Ra}\left(J\left(D_{f}-\frac{1}{\mathrm{Le}}\right)+\frac{\omega^{2} \varepsilon F}{\sigma}\right) \\
& \quad-a^{2} \operatorname{Rs}\left(J-S_{r} J-\omega^{2} F\right)=0, \\
& J^{2}\left(\frac{1}{\mathrm{Le}}+\frac{\varepsilon}{\sigma}\right)+a^{2} \operatorname{Ra}\left(F J\left(D_{f}-\frac{1}{\mathrm{Le}}\right)-\frac{\varepsilon}{\sigma}\right) \\
& \quad-a^{2} \operatorname{Rs}\left(J F-S_{r} J F+1\right)=0 .
\end{aligned}
$$

For stationary convection $\omega=0(n=0)$, we have

$$
\mathrm{Ra}=\frac{\left(\pi^{2}+a^{2}\right)^{2}}{a^{2}}\left(\frac{D_{f} S_{r} \mathrm{Le}-1}{D_{f} \mathrm{Le}-1}\right)+\frac{\left(1-S_{r}\right) \mathrm{Le}}{D_{f} \mathrm{Le}-1} \mathrm{Rs} .
$$

It is clear from (17) that stationary Rayleigh number $\mathrm{Ra}$ is a function of dimensionless wave number $a$, Dufour parameter $D_{f}$, Soret parameter $S_{r}$, Lewis number Le and solutal Rayleigh number Rs, and independent of stress relaxation parameter $F$. Thus for stationary convection the Maxwell viscoelastic fluid behaves like an ordinary Newtonian fluid. This result is the same as obtained by Motsa [24] and Chand and Rana [23].

The critical cell size at the onset of instability is obtained from the condition $(\partial \mathrm{Ra} / \partial a)_{a=a_{c}}=0$, which gives $a_{c}=\pi$.

This result is the same as obtained by Lapwood [4] for Newtonian fluid.

The corresponding critical Rayleigh number $\mathrm{Ra}_{c}$ for steady onset is

$$
\mathrm{Ra}_{c}=4 \pi^{2}\left(\frac{D_{f} S_{r} \mathrm{Le}-1}{D_{f} \mathrm{Le}-1}\right)+\frac{\left(S_{r}-1\right) \mathrm{Le}}{1-D_{f} \mathrm{Le}} \mathrm{Rs} .
$$

This result is the same as obtained by Motsa [24] and Chand and Rana [23].

$$
\begin{aligned}
& \text { If Rs }=D_{f}=S_{r}=0 \text { then } \\
& \qquad \operatorname{Ra}_{c}=4 \pi^{2} .
\end{aligned}
$$

This is exactly the same result which was obtained by Nield [25].

\section{Result and Discussion}

The onset of double diffusive convection in a horizontal layer of Maxwell viscoelastic fluid in the presence of Soret and Dufour in a porous medium is investigated analytically and graphically. The expressions for both the stationary and oscillatory Rayleigh numbers, which characterize the stability of the system, are obtained analytically. The stationary critical Rayleigh number is found to be independent of the viscoelastic parameter $F$; thus Maxwell viscoelastic binary fluid behaves like ordinary Newtonian binary fluid. The stationary critical Rayleigh number and critical wave number are independent of viscoelastic parameter because of the absence of base flow in the present case. The computations are carried out for different values solutal Rayleigh number Rs, Soret parameter $S_{r}$, Dufour parameter $D_{f}$, and Lewis number Le. The parameters considered are in the range of $10^{2} \leq \mathrm{Ra} \leq 10^{5}$ (thermal Rayleigh number), $10^{2} \leq \mathrm{Rs} \leq 10^{3}$ (solutal Rayleigh number), $0 \leq S_{r} \leq 1$ (Soret parameter), $0 \leq D_{f} \leq 1$ (Dufour parameter), $[24,26]$, and $10^{-2} \leq$ Le $\leq 1$ (Lewis number) [27, 28], [29]. We choose the values of Dufour parameter $D_{f}$ and Lewis number Le in such a way that $D_{f}$ Le $\neq 1$.

In order to investigate effects of the Dufour parameter $D_{f}$, Soret parameter $S_{r}$, Lewis number Le, and solutal Rayleigh number Rs on stationary convection, we examine the behavior of $\partial \mathrm{Ra} / \partial D_{f}, \partial \mathrm{Ra} / \partial S_{r}, \partial \mathrm{Ra} / \partial \mathrm{Le}$, and $\partial \mathrm{Ra} / \partial \mathrm{Rs}$ analytically.

From (17), we have

(i)

$$
\begin{aligned}
& \frac{\partial \mathrm{Ra}}{\partial D_{f}}=\frac{\left(1-S_{r}\right) \text { Le }}{\left(D_{f} \text { Le }-1\right)^{2}}\left[\frac{\left(\pi^{2}+a^{2}\right)^{2}}{a^{2}} D_{f}-\text { Le Rs }\right] \\
& \frac{\partial \mathrm{Ra}}{\partial D_{f}}>0 \quad \text { if Le Rs }>\frac{\left(\pi^{2}+a^{2}\right)^{2}}{a^{2}} D_{f} \\
&<0 \quad \text { if Le Rs }<\frac{\left(\pi^{2}+a^{2}\right)^{2}}{a^{2}} D_{f} \\
&=0
\end{aligned}
$$

at two specific values of wave number, which depend upon Lewis number Le, solutal Rayleigh number Rs, and Dufour parameter $D_{f}$ and is independent of Soret parameter $S_{r}$.

Thus for the stationary convection Dufour parameter $D_{f}$ has a stabilizing effect if Le Rs $>\left(\left(\pi^{2}+a^{2}\right)^{2} / a^{2}\right) D_{f}$ and destabilizing effect if Le Rs $<\left(\left(\pi^{2}+a^{2}\right)^{2} / a^{2}\right) D_{f}$ : 
(ii)

$$
\begin{aligned}
\frac{\partial \mathrm{Ra}}{\partial S_{r}} & =\frac{\mathrm{Le}}{D_{f} \mathrm{Le}-1}\left[\frac{\left(\pi^{2}+a^{2}\right)^{2}}{a^{2}} D_{f}-\mathrm{Rs}\right], \\
\frac{\partial \mathrm{Ra}}{\partial S_{r}} & >0 \quad \text { if } \frac{\left(\pi^{2}+a^{2}\right)^{2}}{a^{2}} D_{f}<\mathrm{Rs}, \\
& <0 \quad \text { if } \frac{\left(\pi^{2}+a^{2}\right)^{2}}{a^{2}} D_{f}>\mathrm{Rs}, \\
& =0
\end{aligned}
$$

at two specific values of wave number, which depend upon solutal Rayleigh number Rs and Dufour parameter $D_{f}$ and is independent of Lewis number Le and Soret parameter $S_{r}$.

Thus for stationary convection Soret parameter $S_{r}$, therefore, has a stabilizing effect if $\left(\left(\pi^{2}+a^{2}\right)^{2} / a^{2}\right) D_{f}<$ Rs and destabilizing effect if $\left(\left(\pi^{2}+a^{2}\right)^{2} / a^{2}\right) D_{f}>$ Rs:

(iii)

$$
\begin{aligned}
\frac{\partial \mathrm{Ra}}{\partial \mathrm{Le}} & =\frac{\left(1-S_{r}\right)}{\left(D_{f} \mathrm{Le}-1\right)}\left[\frac{\left(\pi^{2}+a^{2}\right)^{2}}{a^{2}} D_{f}-\mathrm{Rs}\right], \\
\frac{\partial \mathrm{Ra}}{\partial \mathrm{Le}} & >0 \quad \text { if } \frac{\left(\pi^{2}+a^{2}\right)^{2}}{a^{2}} D_{f}<\mathrm{Rs}, \\
& <0 \quad \text { if } \frac{\left(\pi^{2}+a^{2}\right)^{2}}{a^{2}} D_{f}>\mathrm{Rs}, \\
& =0
\end{aligned}
$$

at two specific values of wave number, which depend upon solutal Rayleigh number Rs and Dufour parameter $D_{f}$ and is independent of Lewis number Le and Soret parameter $S_{r}$.

Thus for stationary convection Lewis number Le has a stabilizing effect if $\left(\left(\pi^{2}+a^{2}\right)^{2} / a^{2}\right) D_{f}<$ Rs and destabilizing effect if $\left(\left(\pi^{2}+a^{2}\right)^{2} / a^{2}\right) D_{f}>$ Rs:

(iv)

$$
\frac{\partial \mathrm{Ra}}{\partial \mathrm{Rs}}=\frac{\left(1-S_{r}\right) \mathrm{Le}}{D_{f} \mathrm{Le}-1}<0 .
$$

Thus for stationary convection solutal Rayleigh number Rs has a destabilizing effect.

Now we discussed the effects of various parameters on the onset of double diffusive convection of Maxwell viscoelastic fluid in a porous medium for stationary convection graphically. The convection curves for solutal Rayleigh number Rs, Soret parameter $S_{r}$, Dufour parameter $D_{f}$, and Lewis number Le in the (Ra, $a)$ plane are shown in Figures 1-4.

Figure 1 shows the variation of stationary Rayleigh number with wave number for different values of Dufour parameter and it is found that the Rayleigh number first increases

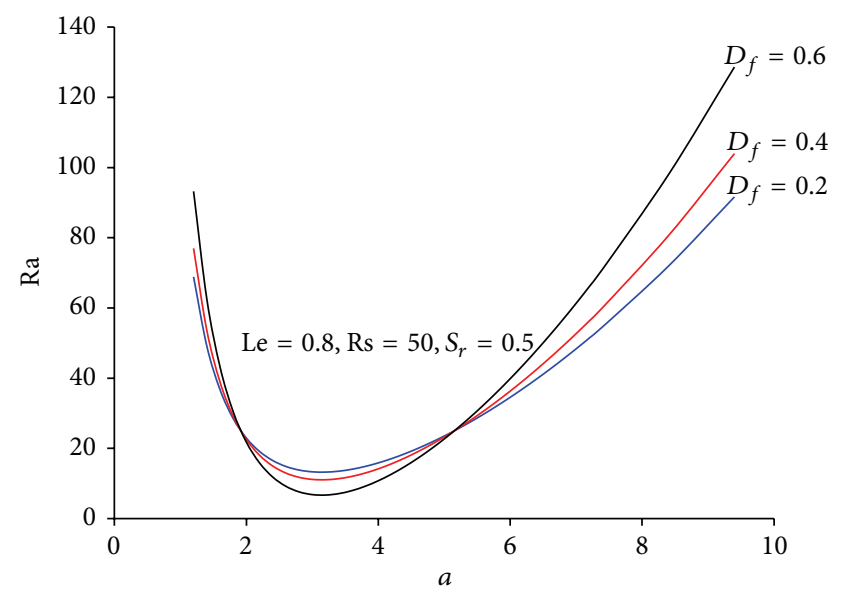

FIGURE 1: Variation of Rayleigh number Ra with wave number $a$ for different values of Dufour parameter $D_{f}$.

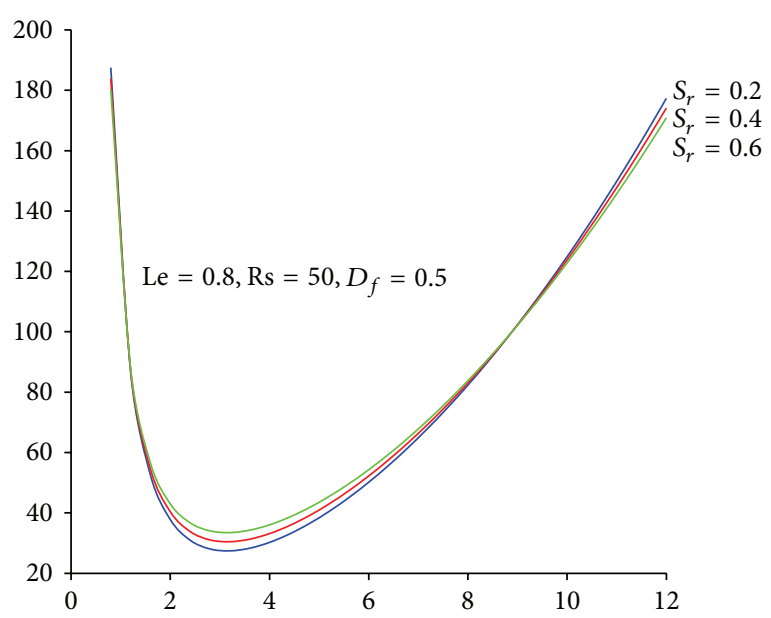

FIGURE 2: Variation of Rayleigh number Ra with wave number $a$ for different values of Soret parameter $S_{r}$.

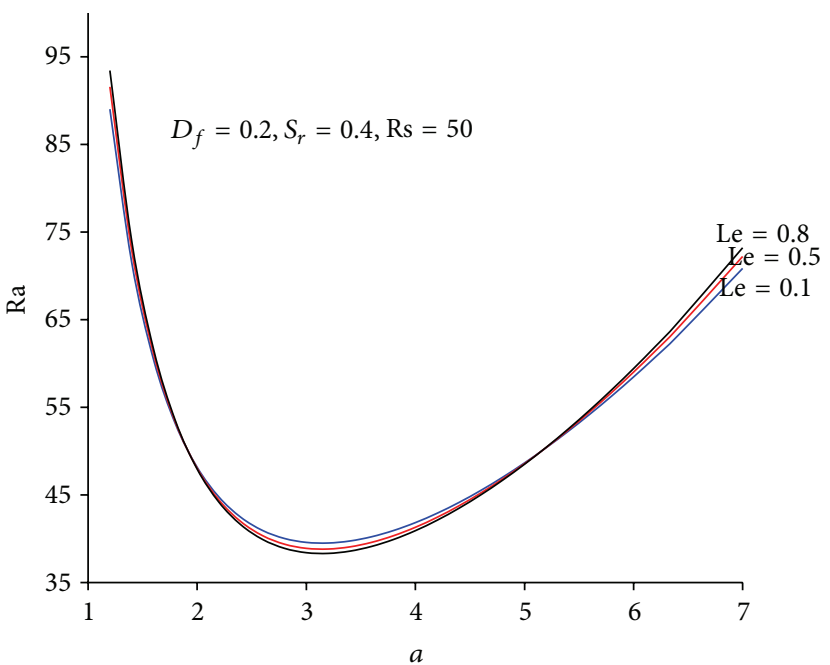

FIgURE 3: Variation of Rayleigh number Ra with wave number $a$ for different values of Lewis number Le. 


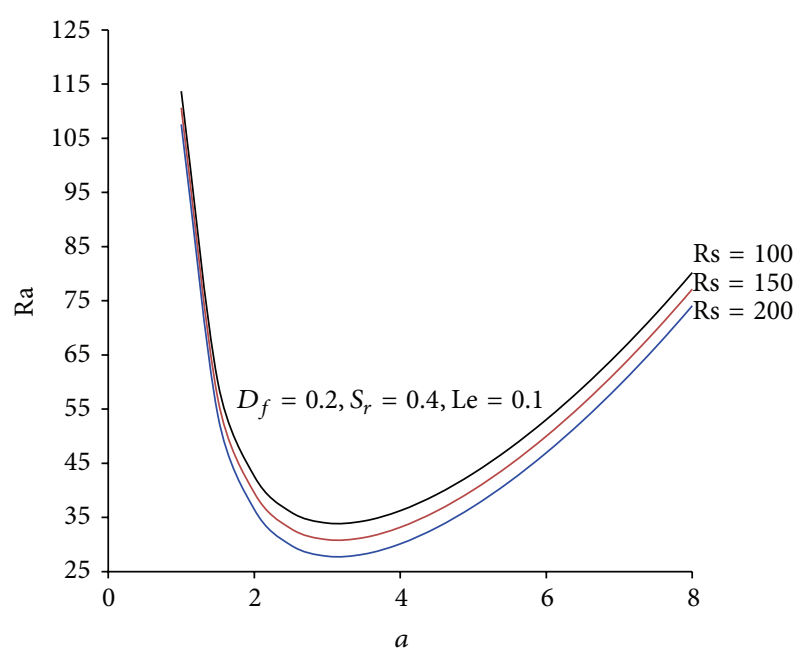

FIGURE 4: Variation of Rayleigh number Ra with wave number $a$ for different values of solutal Rayleigh number Rs.

then decreases and finally increases with increase in the value of Dufour parameter; thus for stationary convection Dufour parameter has both the stabilizing and destabilizing effects depending upon certain conditions.

Figure 2 shows the variation of stationary Rayleigh number with wave number for different values of Soret parameter and it is found that the Rayleigh number first decreases then increases and finally decreases with increase in the value of Soret parameter; thus for stationary convection Soret parameter has both the stabilizing and destabilizing effects depending upon certain conditions.

Figure 3 shows the variation of stationary Rayleigh number with wave number for different values of Lewis number and it is found that the Rayleigh number first increases then decreases and finally increases with increase in the value of Lewis number; thus for stationary convection Lewis number has both the stabilizing and destabilizing effects depending upon certain conditions.

Figure 4 shows the variation of Rayleigh number with wave number for different value of the solutal Rayleigh number Rs and it is found that the Rayleigh number decreases with increase in the value of solutal Rayleigh number Rs; thus solutal Rayleigh number Rs has destabilizing effect on the stationary convection.

Curves in Figures 1-3 intersect two specific values of wave number a which is independent of Lewis number Le and Soret parameter $S_{r}$ as explained in the analytical part of the results and discussion.

\section{Conclusions}

A linear stability analysis of double diffusive convection in a horizontal layer of Maxwell viscoelastic fluid in the presence of Soret and Dufour in a porous medium is investigated analytically and graphically. The expressions for both the stationary and oscillatory Rayleigh numbers, which characterize the stability of the system, are obtained.
The main conclusions are as follows.

(i) In stationary convection Maxwell viscoelastic fluid behaves like ordinary Newtonian fluid.

(ii) Dufour parameter, Soret parameter, and Lewis parameter have both stabilizing and destabilizing effects on the stationary convection.

(iii) Solutal Rayleigh number destabilizes the stationary convection.

(iv) In limiting case when Rs $=S_{r}=D_{f}=0$ the critical thermal Rayleigh number obtained is the same as reported by Nield [25].

\section{Nomenclature}

a: $\quad$ Wave number

C: $\quad$ Solute concentration

$c_{p}: \quad$ Heat capacity

$D_{f}: \quad$ Dufour parameter

$D_{T C}: \quad$ Dufour coefficient

$D_{C T}$ : Soret coefficient

$d: \quad$ Thickness of fluid layer

F: $\quad$ Stress relaxation parameter

g: Acceleration due to gravity

$k_{1}$ : $\quad$ Medium permeability

Le: Lewis number

$n: \quad$ Growth rate of disturbances

p: $\quad$ Pressure

q: $\quad$ Darcy fluid velocity

Ra: Thermal Rayleigh number

$\mathrm{Ra}_{c}: \quad$ Critical Rayleigh number

Rs: $\quad$ Solutal Rayleigh number

$S_{r}: \quad$ Soret parameter

$t: \quad$ Time

$T: \quad$ Temperature

$(u, v, w)$ : Components of fluid velocity

$(x, y, z)$ : Space coordinates.

Greek Symbols

$\rho$ : Density of fluid

$\alpha$ : Coefficient of thermal expansion

$\alpha^{\prime}$ : Analogous solvent coefficient of expansion

$\mu$ : Viscosity

$\kappa$ : Thermal diffusivity

$\kappa^{\prime}$ : Solute diffusivity

$\lambda$ : Relaxation time

$\partial$ : Curly operator

$\varepsilon$ : Porosity

$\omega$ : Dimensionless frequency of oscillation

$\sigma$ : Thermal capacity ratio.

Superscripts

': Nondimensional variables

": Perturbed quantity. 


\section{Subscripts}

0: Value of variables at lower boundary

1: Value of variables at upper boundary

$s$ : Steady state

$f$ : Fluid

$m$ : Porous medium.

\section{Conflict of Interests}

The authors declare that there is no conflict of interests regarding the publication of this paper.

\section{Acknowledgment}

The authors are grateful to the reviewers for their lucid comments and suggestions which have served to improve the research paper.

\section{References}

[1] H. Bénard, "Les tourbillons cellularies dans une nappe liquid," Revue Generale des Sciences Pures et Appliqués, vol. 11, pp. 12611271, 1900.

[2] L. Rayleigh, "On convective currents in a horizontal layer of fluid when the higher temperature is on the under side," Philosophical Magazine, vol. 32, pp. 529-546, 1916.

[3] S. Chandrasekhar, Hydrodynamic and Hydromagnetic Stability, Dover Publication, New York, NY, USA, 1961.

[4] E. R. Lapwood, "Convection of a fluid in porous medium," Mathematical Proceedings of the Cambridge Philosophical Society, vol. 44, no. 4, pp. 508-519, 1948.

[5] R. A. Wooding, "Rayleigh instability of a thermal boundary layer in flow through a porous medium," Journal of Fluid Mechanics, vol. 9, pp. 183-192, 1960.

[6] J. A. M. McDonnel, Cosmic Dust, John Wiley \& Sons, Toronto, Canada, 1978.

[7] D. A. Nield and A. Bejan, Convection in Porous Medium, Springer, New York, NY, USA, 3rd edition, 2006.

[8] O. V. Trevisan and A. Bejan, "Combined heat and mass transfer by natural convection in a porous medium," Advances in Heat Transfer, vol. 20, pp. 315-352, 1990.

[9] M. S. Malashetty and P. Kollur, "The onset of double diffusive convection in a couple stress fluid saturated anisotropic porous layer," Transport in Porous Media, vol. 86, no. 2, pp. 435-459, 2011.

[10] E. Knobloch, "Convection in binary fluids," The Physics of Fluids, vol. 23, no. 9, pp. 1918-1919, 1980.

[11] J. G. Oldroyd, "Non-Newtonian effects in steady motion of some idealized elastico-viscous liquids," Proceedings of the Royal Society A: Mathematical, Physical and Engineering Sciences, vol. 245, pp. 278-297, 1958.

[12] T. Green III, "Oscillating convection in an elasticoviscous liquid," Physics of Fluids, vol. 11, no. 7, pp. 1410-1412, 1968.

[13] C. M. Vest and V. Arpaci, "Overstability of visco -elastic fluid layer heated from below," Journal of Fluid Mechanics, vol. 36, no. 3, pp. 613-623, 1969.

[14] P. K. Bhatia and J. M. Steiner, "Convective instability in a rotating viscoelastic fluid layer," Zeitschrift für Angewandte Mathematik und Mechanik, vol. 52, pp. 321-327, 1972.
[15] P. K. Bhatia and J. M. Steiner, "Thermal instability in a viscoelastic fluid layer in hydromagnetics," Journal of Mathematical Analysis and Applications, vol. 41, pp. 271-283, 1973.

[16] R. C. Sharma and P. Kumar, "Hall efect on thermosolutal instability in a Maxwellian visco-elastic fluid in porous medium," Archives of Mechanics, vol. 48, pp. 199-209, 1996.

[17] K. Prakash and R. Chand, "Thermosolutal instability of Maxwell visco-Elastic fluid with Hall Current, suspended particles and variable gravity in porous medium," Ganita Sandesh, vol. 13, no. 1, pp. 1-12, 1999.

[18] R. Chand and S. K. Kango, "Thermosolutal instability of dusty rotating Maxwell visco-elastic fluid in porous medium," Advances in Applied Science Research, vol. 2, no. 6, pp. 541-553, 2011.

[19] R. Chand, "Gravitational effect on thermal instability of Maxwell visco-elastic fluid in porous medium," Ganita Sandesh, vol. 24, no. 2, pp. 166-170, 2010.

[20] R. Chand, "Effect of suspended particles on thermal instability of Maxwell visco-elastic fluid with variable gravity in porous medium," Antarctica Journal of Mathematics, vol. 8, no. 6, pp. 487-497, 2011

[21] R. Chand, "Thermal instability of rotating Maxwell visco-elastic fluid with variable gravity in porous medium," The Journal of the Indian Mathematical Society, vol. 80, no. 1-2, pp. 23-31, 2013.

[22] R. Chand and A. Kumar, "Thermal instability of rotating Maxwell visco-elastic fluid with variable gravity in porous medium," International Journal of Advances in Applied Mathematics and Mechanics, vol. 1, no. 2, pp. 30-38, 2013.

[23] R. Chand and G. C. Rana, "Dufour and soret effects on the thermosolutal instability of rivlin-ericksen elastico-viscous fluid in porous medium," Zeitschrift fur Naturforschung A, vol. 67, no. 12, pp. 685-691, 2012.

[24] S. S. Motsa, "On the onset of convection in a porous layer in the presence of Dufour and Soret effects," SAMSA Journal of Pure and Applied Mathematics, vol. 3, pp. 58-65, 2008.

[25] D. A. Nield, "Convection in a porous medium with inclined temperature gradient," International Journal of Heat and Mass Transfer, vol. 34, no. 1, pp. 87-92, 1991.

[26] N. Nithyadevi and R. J. Yang, "Double diffusive natural convection in a partially heated enclosure with Soret and Dufour effects," International Journal of Heat and Fluid Flow, vol. 30, no. 5, pp. 902-910, 2009.

[27] J. Martínez-Mardones, R. Tiemann, and D. Walgraef, "Convection in binary viscoelastic fluid," Revista Mexicana de Fisica, vol. 48, no. 3, pp. 103-105, 2002.

[28] Z. Yang, S. Wang, M. Zhao, S. Li, and Q. Zhang, "The onset of double diffusive convection in a viscoelastic fluid-saturated porous layer with non-equilibrium model," PLoS ONE, vol. 8, no. 11, Article ID e79956, 2013.

[29] D. Srinivasacharya and G. Swamy Reddy, "Double diffusive natural convection in power-law fluid saturated porous medium with Soret and Dufour effects," Journal of the Brazilian Society of Mechanical Sciences and Engineering, vol. 34, no. 4, pp. 525-530, 2012 . 

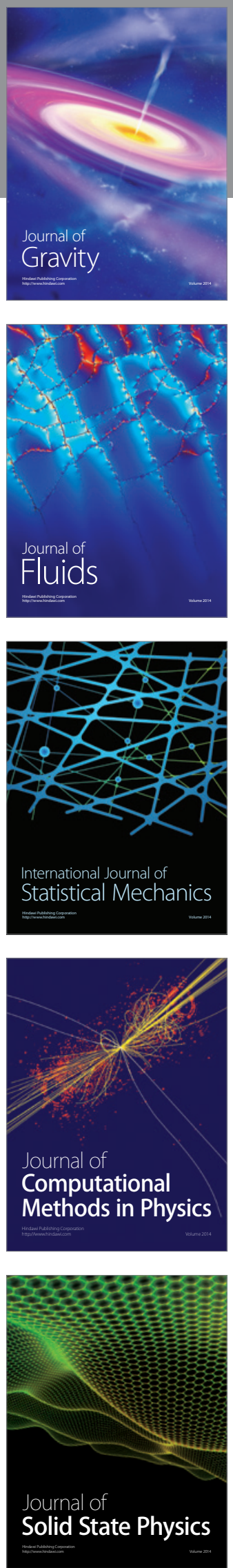

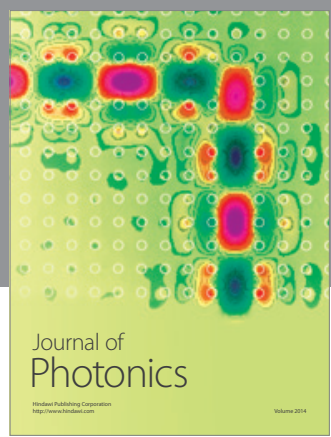

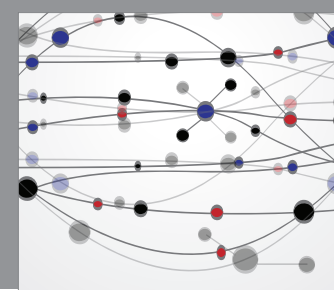

The Scientific World Journal

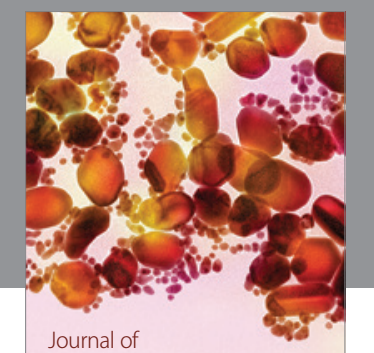

Soft Matter
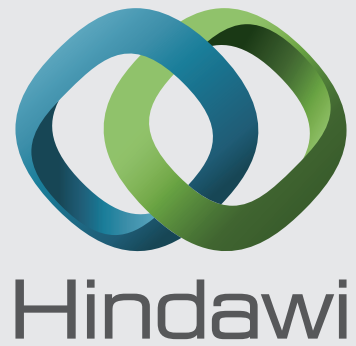

Submit your manuscripts at

http://www.hindawi.com
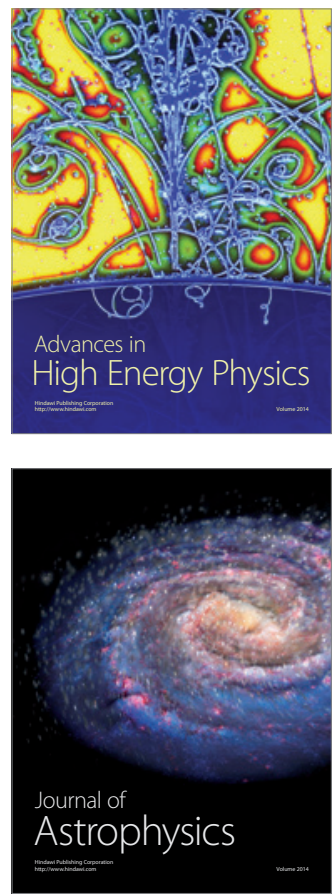
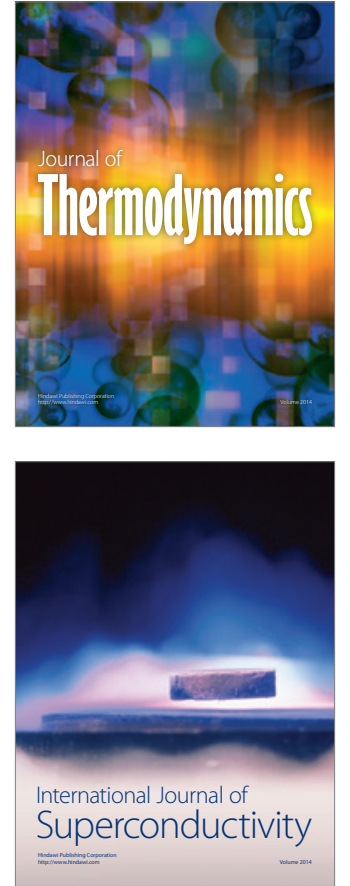
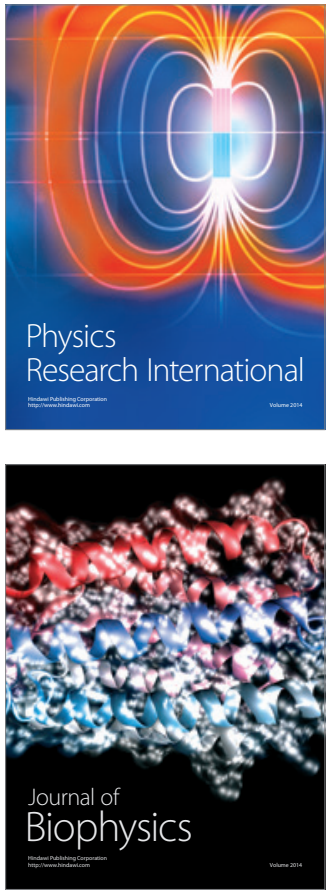
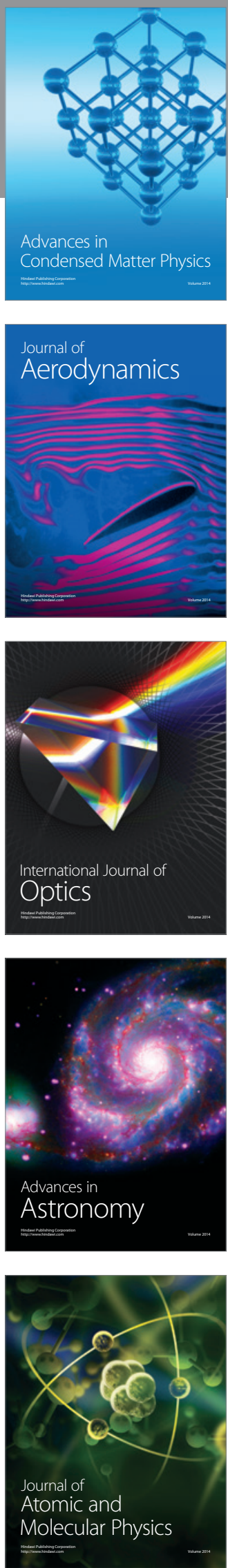